\title{
THE APS-INDEX AND THE SPECTRAL FLOW
}

\author{
KoEn VAn DEN Dungen And LEnNART RongE*
}

\begin{abstract}
We study the Atiyah-Patodi-Singer (APS) index, and its equality to the spectral flow, in an abstract, functional analytic setting. More precisely, we consider a (suitably continuous or differentiable) family of self-adjoint Fredholm operators $A(t)$ on a Hilbert space, parametrised by $t$ in a finite interval. We then consider two different operators, namely $D:=\frac{d}{d t}+A$ (the abstract analogue of a Riemannian Dirac operator) and $D:=\frac{d}{d t}-i A$ (the abstract analogue of a Lorentzian Dirac operator). The latter case is inspired by a recent index theorem by Bär and Strohmaier (Amer. J. Math. 141 (2019), 1421-1455) for a Lorentzian Dirac operator equipped with APS boundary conditions. In both cases, we prove that the Fredholm index of the operator $D$ equipped with APS boundary conditions is equal to the spectral flow of the family $A(t)$.
\end{abstract}

Mathematics subject classification (2020): 47A53, 58J20, 58J30.

Keywords and phrases: Atiyah-Patodi-Singer index, Fredholm pairs of projections, spectral flow.

\section{REFERENCES}

[1] M. F. ATIYAh, V. K. PATOdi, AND I. M. Singer, Spectral asymmetry and Riemannian geometry, I, Math. Proc. Cambridge Philos. Soc. 77 (1975), 43-69.

[2] M. F. AtiYah, V. K. Patodi, AND I. M. Singer, Spectral asymmetry and Riemannian geometry, III, Math. Proc. Cambridge Philos. Soc. 79 (1976), 71-99.

[3] J. AVRon, R. SeILER, AND B. Simon, The index of a pair of projections, J. Funct. Anal. 120 (1994), no. $1,220-237$.

[4] S. AzZali And C. WAHL, Spectral flow, index and the signature operator, J. Topol. Anal. 03 (2011), no. 01, 37-67.

[5] C. BÄR And A. STrohmaier, An index theorem for Lorentzian manifolds with compact spacelike Cauchy boundary, Amer. J. Math. 141 (2019), no. 5, 1421-1455.

[6] A. N. BERNAL AND M. SÁNCHEZ, Smoothness of time functions and the metric splitting of globally hyperbolic space-times, Commun. Math. Phys. 257 (2005), 43-50.

[7] M. BRAVERMAn, An index of strongly Callias operators on Lorentzian manifolds with non-compact boundary, Math. Z. 294 (2020), 229-250.

[8] J. BRÜNING AND M. LeSCH, On boundary value problems for Dirac type operators, J. Funct. Anal. 185 (2001), no. 1, 1-62.

[9] U. BUnKe AND T. HiRsChmAnn, The index of the scattering operator on the positive spectral subspace, Commun. Math. Phys. 148 (1992), 487-502.

[10] A. L. Carey, J. Phillips, And A. Rennie, A noncommutative Atiyah-Patodi-Singer index theorem in KK-theory, J. Reine Angew. Math. 643 (2010), 59-109.

[11] J. Gell-Redman, N. Haber, And A. VASY, The Feynman propagator on perturbations of Minkowski space, Commun. Math. Phys. 342 (2016), 333-384.

[12] E. Hille AND R. Phillips, Functional analysis and semi-groups, Colloquium publications, vol. 31, American Mathematical Society, 1996.

[13] J. KAAD AND M. LESCH, Spectral flow and the unbounded Kasparov product, Adv. Math. 248 (2013), 495-530.

[14] T. Kato, Perturbation theory for linear operators, corrected printing, second ed., Classics in Mathematics, Springer-Verlag, 1980. 
[15] M. LESCH, The uniqueness of the spectral flow on spaces of unbounded self-adjoint Fredholm operators, Spectral geometry of manifolds with boundary and decomposition of manifolds (B. BoossBavnbek, G. Grubb, and K. P. Wojciechowski, eds.), Contemp. Math., vol. 366, Amer. Math. Soc., 2005, pp. 193-224.

[16] A. PAZY, Semigroups of linear operators and applications to partial differential equations, Applied Mathematical Sciences, vol. 44, Springer, New York, 1983.

[17] J. PHILlips, Self-adjoint Fredholm operators and spectral flow, Canad. Math. Bull. 39 (1996), no. 4, $460-467$.

[18] J. Robbin And D. Salamon, The spectral flow and the Maslov index, Bull. Lond. Math. Soc. 27 (1995), no. 1, 1-33.

[19] L. RonGE, Index theory for globally hyperbolic spacetimes, Master's thesis, University of Bonn, 2019, arXiv: 1910.10452.

[20] M. StAROSTKA AND N. WATERSTRAAT, On a comparison principle and the uniqueness of spectral flow.

[21] K. VAn Den Dungen, Families of spectral triples and foliations of space (time), J. Math. Phys. 59 (2018), no. 6, 063507.

[22] K. VAn Den Dungen, The index of generalised Dirac-Schrödinger operators, J. Spectr. Theory 9 (2019), 1459-1506. 